\title{
Analysis of Fractional Order Chaotic Financial Model with Minimum Interest Rate Impact
}

\author{
Muhammad Farman $^{1}$, Ali Akgüi ${ }^{2, *} \mathbb{C}$, Dumitru Baleanu ${ }^{3,4,5}$, Sumaiyah Imtiaz ${ }^{1}$ \\ and Aqeel Ahmad ${ }^{1}$ \\ 1 Department of Mathematics and Statistics, University of Lahore, Lahore 54590, Pakistan; \\ farmanlink@gmail.com (M.F.); sumaiyah.imtiaz88@gmail.com (S.I.); aqeelahmad.740@gmail.com (A.A.) \\ 2 Art and Science Faculty, Department of Mathematics, Siirt University, Siirt 56100, Turkey \\ 3 Department of Mathematics, Cankaya University, Ankara 06530, Turkey; dumitru.baleanu@gmail.com \\ 4 Institute of Space Sciences, R76900 Magurele-Bucharest, Romania \\ 5 Department of Medical Research, China Medical University, Taichung 40402, Taiwan \\ * Correspondence: aliakgul00727@gmail.com
}

Received: 19 July 2020; Accepted: 14 August 2020; Published: 21 August 2020

check for updates

\begin{abstract}
The main objective of this paper is to construct and test fractional order derivatives for the management and simulation of a fractional order disorderly finance system. In the developed system, we add the critical minimum interest rate $d$ parameter in order to develop a new stable financial model. The new emerging paradigm increases the demand for innovation, which is the gateway to the knowledge economy. The derivatives are characterized in the Caputo fractional order derivative and Atangana-Baleanu derivative. We prove the existence and uniqueness of the solutions with fixed point theorem and an iterative scheme. The interest rate begins to rise according to initial conditions as investment demand and price exponent begin to fall, which shows the financial system's actual macroeconomic behavior. Specifically component of its application to the large scale and smaller scale forms, just as the utilization of specific strategies and instruments such fractal stochastic procedures and expectation.
\end{abstract}

Keywords: chaotic finance; fractional calculus; Atangana-Baleanu derivative; uniqueness of the solution; fixed point theory

\section{Introduction}

Different parts of the financial sector are investigated through mathematical models, this article is helpful in discussing advantages and drawbacks of mathematical models in the financial associations. Different solutions for improving mathematical models and obstructions in the application zone is also discussed. Mathematical modeling is the technique in which sensible and similar numerical expressions from vertical frameworks are made which is used in translating different issues including drawing systematic ideas. These systematic ideas are used in formulating strategy by choosing information and understanding problem [1]. Mathematical models help in different fields of science such as sociology and engineering. Mathematics have an important place in the field of finance. In the field of finance, account related theory on assessment of exercises on the money related administrators [2]. Different parts of financial market types and different scientific and numerical systems are drawn by using mathematical models [3].

Different types of genuine certified systems are derived through the device of fractional calculus [4]. We establish some writing with the help of the research work and construct the hypothesis of fractional calculus and exhibiting several utilizations. The recently presented Caputo Fabrizio fractional derivative is used to examine the partial model of an adjusted Kawahara condition by 
Kumar et al. [5]. The Atangana-Baleanu partial administrator is used to formulate fragmentary augmentation of a regularized long wave condition [6]. Arrangement of the fragmentary control issues including a Mittag-Leffler non-specific piece is proposed by Baleanu et al. [7]. In another work, the development of fractional analysis has been given in [8]. Fractional calculus is used by the scientist named Jajarmi for separating a hyperchaotic financial system [9]. The fractional calculus operatives including different functions have been derived effectively for mathematical modeling of various complex issues in changed areas of science and engineering, for example, liquid elements, plasma material science, astronomy, picture handling, stochastic dynamical framework, and controlled atomic combination [1,10-12]. Recently, Caputo and Fabrizio [13] derived another fractional derivative for some engineering and thermo dynamical systems, and this new derivation is better than the old style of Caputo derivative. Another fractional derivative for observing the possibility of Fisher's response dispersion condition is derived by Atangana [14]. In this article [15], by using these two approaches, we have defined a new contraction in one of the most extended abstract spaces known. In [16], we have demonstrated a novel approximate-analytical solution method, which is called the Laplace homotopy analysis method (LHAM) using the Caputo-Fabrizio fractional derivative operator. We investigated in [17] existence and uniqueness conditions of solutions of a nonlinear differential equation containing the Caputo-Fabrizio operator in Banach spaces. A solution method is coupled with a kind of integral transformation, namely the Elzaki transform, and apply it to two different nonlinear regularized long wave equations in [18]. In [19], time-fractional partial differential equations (FPDEs) involving singular and non-singular kernel are considered. For more details, see [20-22].

In this paper, we need to use fractional parameters using the Caputo and $\mathrm{ABC}$ derivatives method with fractional derivatives to build the model of complex nonlinear differential equations. Complex financial system models of complex actions provide a new perspective as a result of patterns and actual behavior of the financial system's internal structure.

\section{Preliminaries}

The fractional derivative of Liouville Caputo $[13,23]$ is presented as

$$
{ }_{t_{0}}^{C} D_{t}^{\kappa}\{g(t)\}=\frac{1}{\Gamma(1-\kappa)} \int_{t_{0}}^{t} \frac{d}{d t} g(\psi)(t-\psi)^{-\kappa} d \psi,
$$

where $\Gamma($.$) refers to the function of Gamma. Laplace transform of the above derivative is obtained$ as $[23,24]$ :

$$
\mathcal{L}\left\{{ }_{0}^{C} D_{t}^{\kappa}\{g(t)\}\right\}(s)=S^{\kappa} G(S)-\sum_{k=0}^{m-1} S^{\kappa-k-1} g^{(k)}(0)
$$

Recently, Atangana and Baleanu proposed a fractional derivative with the Mittag-Leffler function as the kernel of differentiation. This kernel is non-singular and nonlocal and preserves the benefits of the above Liouville-Caputo derivative. The Atangana-Baleanu derivative has been defined as [25]:

$$
{ }_{t_{0}}^{A B C} D_{t}^{\kappa}\{g(t)\}=\frac{Z(\kappa)}{1-\kappa} \int_{t_{0}}^{t} \frac{d}{d t} g(\psi) E_{\kappa}\left[-\kappa \frac{(t-\psi)^{\kappa}}{1-\kappa}\right] d \psi, n-1<\kappa(t) \leq n,
$$

where $\kappa \epsilon \Re, Z(\kappa)$ refers to the $Z(0)=Z(1)=1$ and $E \kappa($.$) refers to the equation Mittag-Leffler.$ Equation (3) Laplace is defined as follows:

$$
\mathcal{L}\left\{\begin{array}{l}
A B C \\
0
\end{array} D_{t}^{\kappa}\{g(t)\}\right\}(s)=\frac{Z(\kappa)}{1-\kappa} \mathcal{L}\left[\int_{\kappa}^{t} \frac{d}{d t} g(\psi) E_{\kappa}\left[-\kappa \frac{(t-\psi)^{\kappa}}{1-\kappa}\right] d \psi\right](s)=\frac{Z(\kappa)}{1-\kappa} \frac{s^{\kappa} \mathcal{L}[g(t)](s)-s^{\kappa-1} g(0)}{s^{\kappa}+\frac{\kappa}{1-\kappa}}
$$


The fractional integral associated with the Atangana-Baleanu derivative with non-local kernel is defined as

$$
{ }_{t_{0}}^{A B} D_{t}^{\kappa}\{g(t)\}=\frac{1-\kappa}{Z(\kappa)} g(t)+\frac{\kappa}{Z(\kappa) \Gamma(\kappa)} \int_{t_{0}}^{t} g(\psi)(t-\psi)^{\kappa-1} d \psi
$$

When $\kappa$ is equivalent to zero, the initial function will be retrieved. $\kappa=1$ will be retrieved from the classical ordinary integral.

\section{Liouville-Caputo Sense}

The strategy is an experimental system dependent on the blend of homotopy analysis technique and Laplace's transformation with polynomial homotopy [23,26]. The primary steps of this strategy are characterized as follows:

Step 1. We should take a look at the following condition:

$$
D_{t}^{\kappa}\{g(h, t)\}+\Xi[h] g(h, t)+\wedge[h] g(h, t)=\eta(h, t), \quad t>0, \quad h \in \Re, \quad 0<\kappa \leq 1,
$$

where $\Xi[h]$ is a bounded linear operator in $h$. While the nonlinear operator $\wedge[h]$ in $\mathrm{h}$ is Lipschitz continuous and satisfying $|\wedge(g)-\wedge(\phi)| \leq \theta|g-\phi|$, where $\theta>0$ and $\eta(h, t)$ is a continuous function. The boundary and initial conditions can be treated in a similar way.

Step 2. Applying the methodology proposed in $[23,27]$, we get the following $\mathrm{m}$-th order deformation equation:

$$
\begin{array}{r}
g_{m}(h, t)=\left(X_{m}+\hbar\right) g_{m-1}-\hbar\left(1-X_{m}\right) \sum_{i=0}^{j-1} t^{i} g^{(i-1)}(0) \\
+\hbar \mathcal{L}^{-1}\left(\frac{1}{S^{\kappa}} \mathcal{L}\left(\Xi_{m-1}[h] g_{m-1}(h)+\sum_{k=0}^{m-1} P_{k}\left(g_{0}, g_{1}, \ldots, g_{m}\right)-\Psi(h, t)\right)\right)
\end{array}
$$

where the Laplace transform is implemented in Caputo sense (1) and $P_{k}$ is the homotopy polynomial described by Odibat in [28].

Step 3. Regarding homotopy polynomials, the nonlinear term $\wedge[h] g(h, t)$ is extended as

$$
\wedge[g(h, t)]=\wedge\left(\sum_{k=0}^{m-1} g_{m}(h, t)\right)=\sum_{m=0}^{\infty} P_{m} g^{m}
$$

Step 4. Expanding the nonlinear term in (6) as a progression of polynomials for homotopy, we can compute the diverse $g_{m}(h, t)$ for $m>1$ and Equation solutions (5) can be written as

$$
g(h, t)=\sum_{\infty}^{m=0} g_{m}(h, t)
$$

The classical form of the model first studied in [29] and we modify the model by adding $\mathrm{d}$ as critical minimum interest rate. By using this methodology, a Liouville-Caputo fractional order derivative was utilized to solve the using time-fractional funding model:

$$
\begin{gathered}
{ }_{0}^{C} D_{t}^{\kappa} x(t)=z(t)+x(t) y(t)-a x(t), \\
{ }_{0}^{C} D_{t}^{\kappa} y(t)=1-b y(t)-x(t) x(t), \\
{ }_{0}^{C} D_{t}^{\kappa} z(t)=d-x(t)-c z(t),
\end{gathered}
$$


where $x, y$, and $z$ are the state variables representing interest rate, investment demand, and price index, respectively, and we add the critical minimum interest rate $d$ parameter in [30]. The parameter $a$ is for savings, $b$ is to cost per investment and $c$ is the elasticity of market demand, although the parameters are non-negative constants i.e., $a=3, b=0.1$ and $c=1$.

From the above model (10)-(12), we use the parameter $d$ to modify the model, where $d$ represents critical minimum interest rate with initial conditions $x(0)=n_{1}=0.1, y(0)=n_{2}=4, z(0)=n_{3}=0.5$.

Solution. We also implemented the Laplace transform (2) to the system's first formula on (10):

$$
s^{\kappa} \bar{x}(s)-s^{\kappa-1} x(0)=\mathcal{L}\{z(t)+x(t) y(t)-a x(t)\}
$$

The initial conditions are taken and the above equation is simplified

$$
\bar{x}(s)=\frac{x(0)}{s}+\mathcal{L}\{z(t)+x(t) y(t)-a x(t)\}
$$

With inverse Laplace transform to Equation (14), getting

$$
x(t)=n_{1}+\mathcal{L}^{-1}\left[\frac{1}{\mathcal{S}^{\kappa}} \mathcal{L}\{z(t)+x(t) y(t)-a x(t)\}\right],
$$

For the other equations shown in Equations (11) and (12), we get

$$
\begin{gathered}
y(t)=n_{2}+\frac{t^{\kappa}}{\Gamma(\kappa+1)}-\mathcal{L}^{-1} \frac{1}{s^{\kappa}} \mathcal{L}\{b y(t)+x(t) x(t)\}, \\
z(t)=n_{3}+\frac{d t^{\kappa}}{\Gamma(\kappa+1)}-\mathcal{L}^{-1} \frac{1}{s^{\kappa}} \mathcal{L}\{x(t)+c z(t)\}, \\
{[\mathfrak{j}(\mathfrak{t} ; \mathfrak{p})]=\mathcal{L}\left[\phi_{j}(t ; p)\right], j=1,2,3}
\end{gathered}
$$

with feature $(\mathfrak{e})=0$ where $\mathrm{e}$ is constant. Let's describe the following system as:

$$
\begin{gathered}
N\left[\phi_{1}(t ; p)\right]=\mathcal{L}\left[\phi_{1}(t ; p)\right]-n_{1}+\frac{1}{s^{\kappa}} \mathcal{L}\left\{\phi_{3}+\phi_{1} \phi_{2}-a \phi_{1}\right\} \\
N\left[\phi_{2}(t ; p)\right]=\mathcal{L}\left[\phi_{2}(t ; p)\right]-n_{2}-\frac{1}{s^{\kappa}} \mathcal{L}\left\{b \phi_{2}+\phi_{1} \phi_{1}\right\} \\
N\left[\phi_{3}(t ; p)\right]=\mathcal{L}\left[\phi_{3}(t ; p)\right]-n_{3}-\frac{1}{s^{\kappa}} \mathcal{L}\left\{\phi_{1}+c \phi_{3}\right\}
\end{gathered}
$$

The equation of so-called zero-order deformation is given by

$$
(1-p)\left[\mathfrak{j}(\mathfrak{t} ; \mathfrak{p})-\mathfrak{u}_{\mathcal{o}}(\mathfrak{t})\right]=p \hbar\left[\phi_{j}(t ; p)\right], j=1,2,3
$$

when $p=0$ and $p=1$, we have

$$
\phi_{j}(t ; 0)=u_{0}(t), \phi_{j}(t ; 1)=u(t), j=1,2,3
$$

The deformation equations of the mth-order are given

$$
\mathcal{L}\left\{x_{m}(t)-P_{m} x_{m-1}(t)\right\}=\hbar S_{m}\left(x_{m-1}, t\right)
$$




$$
\begin{aligned}
& \mathcal{L}\left\{y_{m}(t)-P_{m} y_{m-1}(t)\right\}=\hbar S_{m}\left(y_{m-1}, t\right) \\
& \mathcal{L}\left\{z_{m}(t)-P_{m} z_{m-1}(t)\right\}=\hbar S_{m}\left(z_{m-1}, t\right)
\end{aligned}
$$

Transforming the inverse Laplace into Equations (24)-(26). We've got this

$$
\begin{aligned}
& x_{m}(t)=P_{m} x_{m-1}(t)+\hbar S_{m}\left(x_{m-1}, t\right) \\
& y_{m}(t)=P_{m} y_{m-1}(t)+\hbar S_{m}\left(y_{m-1}, t\right) \\
& z_{m}(t)=P_{m} z_{m-1}(t)+\hbar S_{m}\left(z_{m-1}, t\right)
\end{aligned}
$$

where

$$
\begin{gathered}
S_{m}\left(x_{m-1}^{\rightarrow}, t\right)=\mathcal{L}\left[x_{m-1}(t)\right]-\left(1-P_{m}\right)\left(n_{1}+\frac{1}{s^{\kappa}} \mathcal{L}\left\{z_{m-1}+H_{m}-a \cdot x_{m-1}\right\}\right) \\
S_{m}\left(y_{m-1}, t\right)=\mathcal{L}\left[y_{m-1}(t)\right]-\left(1-P_{m}\right)\left(n_{2}+\frac{t^{\kappa}}{\Gamma(\kappa+1)}-\frac{1}{s^{\kappa}} \mathcal{L}\left\{b \cdot y_{m-1}+K_{m}\right\}\right) \\
S_{m}\left(z_{m-1}, t\right)=\mathcal{L}\left[z_{m-1}(t)\right]-\left(1-P_{m}\right)\left(n_{3}+\frac{d t^{\kappa}}{\Gamma(\kappa+1)}-\frac{1}{s^{\kappa}} \mathcal{L}\left\{x_{m-1}+c . z_{m-1}\right\}\right)
\end{gathered}
$$

The mth-order deformation equation solution (24)-(26) is presented as:

$$
\begin{gathered}
x_{m}(t)=\left(P_{m}+\hbar\right) x_{m-1}-\hbar\left(1-P_{m}\right)\left(n_{1}\right)+\hbar \mathcal{L}^{-1}\left\{\frac{1}{s^{\kappa}} \mathcal{L}\left\{z_{m-1}+H_{m}-a \cdot x_{m-1}\right\}\right\} \\
y_{m}(t)=\left(P_{m}+\hbar\right) y_{m-1}-\hbar\left(1-P_{m}\right)\left(n_{2}+\frac{t^{\kappa}}{\Gamma(\kappa+1)}\right)-\hbar \mathcal{L}^{-1}\left\{\frac{1}{s^{\kappa}} \mathcal{L}\left\{b \cdot y_{m-1}+K_{m}\right\}\right\} \\
z_{m}(t)=\left(P_{m}+\hbar\right) z_{m-1}-\hbar\left(1-P_{m}\right)\left(n_{3}+\frac{d t^{\kappa}}{\Gamma(\kappa+1)}\right)-\hbar \mathcal{L}^{-1}\left\{\frac{1}{s^{\kappa}} \mathcal{L}\left\{x_{m-1}+c . z_{m-1}\right\}\right\}
\end{gathered}
$$

where

$$
\begin{aligned}
& H_{m}=\frac{1}{\Gamma m+1}\left[\frac{d^{m}}{d p^{m}} N\left[\left(p \phi_{1}(t ; p)\right)\left(p \phi_{2}(t ; p)\right)\right]\right]_{p=0^{\prime}} \\
& K_{m}=\frac{1}{\Gamma m+1}\left[\frac{d^{m}}{d p^{m}} N\left[\left(p \phi_{1}(t ; p)\right)\left(p \phi_{1}(t ; p)\right)\right]\right]_{p=0^{\prime}}
\end{aligned}
$$

Finally, the solutions of the Equations (10)-(12) are

$$
\begin{aligned}
& x(t)=x_{0}(t)+x_{1}(t)+x_{2}(t)+\ldots \ldots .=\sum_{m=0}^{\infty} x_{m}(t) \\
& y(t)=y_{0}(t)+y_{1}(t)+y_{2}(t)+\ldots \ldots .=\sum_{m=0}^{\infty} y_{m}(t)
\end{aligned}
$$




$$
z(t)=z_{0}(t)+z_{1}(t)+z_{2}(t)+\ldots \ldots=\sum_{m=0}^{\infty} z_{m}(t)
$$

Through combining the Laplace transform (2) and its inverse, another model (10)-(12) solution can be obtained. The iterative scheme is given through

$$
\begin{gathered}
x_{n}(t)=n_{1}+\mathcal{L}^{-1}\left\{\frac{1}{s^{\mathcal{K}}} \mathcal{L}\left\{z_{n-1}(t)+x_{n-1}(t) \cdot y_{n-1}(t)-a \cdot x_{n-1}(t)\right\}(s)\right\}(t) \\
y_{n}(t)=n_{2}+\mathcal{L}^{-1}\left\{\frac{1}{s^{\kappa}} \mathcal{L}\left\{1-b \cdot y_{n-1}(t)-x_{n-1}(t) \cdot x_{n-1}(t)\right\}(s)\right\}(t) \\
z_{n}(t)=n_{3}+\mathcal{L}^{-1}\left\{\frac{1}{s^{\mathcal{L}}} \mathcal{L}\left\{d-x_{n-1}(t)-c \cdot z_{n-1}(t)\right\}(s)\right\}(t)
\end{gathered}
$$

where $n_{1}, n_{2}$, and $n_{3}$ are the initial conditions. If $n$ tends to infinity, it is assumed that the solution is a limit

$$
x(t)=\lim _{n \rightarrow \infty} x_{n}(t), y(t)=\lim _{n \rightarrow \infty} y_{n}(t), z(t)=\lim _{n \rightarrow \infty} z_{n}(t)
$$

Theorem 1. Equations recursive forms (41)-(43) are stable.

Proof. We are going to assume the following. There are five positive constants D, E, and F can be found such that for all $0 \leq t \leq T \leq \infty$,

$$
\|x(t)\|<D ;\|y(t)\|<E ;\|z(t)\|<F
$$

Now, we consider a subset of $L_{2}((e, f)(0, T))$ defined as follows:

$$
\Xi=\left\{\beta:(e, f)(0, T) \rightarrow \Xi, \frac{1}{\Gamma(\kappa)} \int(t-\beta)^{(\kappa-1)} v(\beta) u(\beta) d \beta<\infty\right\}
$$

We have

$$
\begin{array}{r}
\vartheta(x, y, z)=z(t)+x(t) y(t)-a x(t) \\
=1-b y(t)+x(t) x(t) \\
=d-x(t)-c z(t)
\end{array}
$$

Then,

$$
\begin{array}{r}
=<\vartheta(x, y, z)-\vartheta\left(x_{1}, y_{1}, z_{1}\right),\left(x-x_{1}, y-y_{1}, z-z_{1}\right)> \\
<\left(z(t)-z_{1}(t)\right)+\left(x(t)-x_{1}(t)\right)\left(y(t)-y_{1}(t)\right)-a\left(x(t)-x_{1}(t)\right),\left(x(t)-x_{1}(t)\right)> \\
<1-b\left(y(t)-y_{1}(t)\right)+\left(x(t)-x_{1}(t)\right)\left(x(t)-x_{1}(t)\right),\left(y(t)-y_{1}(t)\right)>, \\
<d-\left(x(t)-x_{1}(t)\right)-c\left(z(t)-z_{1}(t)\right),\left(z(t)-z_{1}(t)\right)>,
\end{array}
$$

where

$$
x(t) \neq x_{1}(t) ; y(t) \neq y_{1}(t) ; z(t) \neq z_{1}(t)
$$


We obtain

$$
\begin{array}{r}
\quad<\vartheta(x, y, z)-\vartheta\left(x_{1}, y_{1}, z_{1}\right),\left(x-x_{1}, y-y_{1}, z-z_{1}\right)>, \\
<\left\{\frac{\left\|z(t)-z_{1}(t)\right\|}{\left\|x(t)-x_{1}(t)\right\|}+\left\|y(t)-y_{1}(t)\right\|-a\right\}\left\|x(t)-x_{1}(t)\right\|^{2}, \\
<\left\{\frac{1}{\left\|y(t)-y_{1}(t)\right\|}-b-\frac{\left\|x(t)-x_{1}(t)\right\|^{2}}{\left\|y(t)-y_{1}(t)\right\|}\right\}\left\|y(t)-y_{1}(t)\right\|^{2}, \\
<\left\{\frac{d}{\left\|z(t)-z_{1}(t)\right\|}+\frac{\left\|x(t)-x_{1}(t)\right\|}{\left\|z(t)-z_{1}(t)\right\|}-c\right\}\left\|z(t)-z_{1}(t)\right\|^{2},
\end{array}
$$

where

$$
\begin{array}{r}
<\vartheta(x, y, z)-\vartheta\left(x_{1}, y_{1}, z_{1}\right),\left(x-x_{1}, y-y_{1}, z-z_{1}\right)> \\
<A\left\|x(t)-x_{1}(t)\right\|^{2}, \\
<B\left\|y(t)-y_{1}(t)\right\|^{2}, \\
<C\left\|z(t)-z_{1}(t)\right\|^{2},
\end{array}
$$

with

$$
\begin{gathered}
A=\frac{\left\|z(t)-z_{1}(t)\right\|}{\left\|x(t)-x_{1}(t)\right\|}+\left\|y(t)-y_{1}(t)\right\|-a \\
B=\frac{1}{\left\|y(t)-y_{1}(t)\right\|}-b-\frac{\left\|x(t)-x_{1}(t)\right\|^{2}}{\left\|y(t)-y_{1}(t)\right\|} \\
C=\frac{d}{\left\|z(t)-z_{1}(t)\right\|}+\frac{\left\|x(t)-x_{1}(t)\right\|}{\left\|z(t)-z_{1}(t)\right\|}-c
\end{gathered}
$$

Additionally, if we find a non-null vector $\left(x_{1}, y_{1}, z_{1}\right)$ using a certain routine as above, we get

$$
\begin{aligned}
& <A\left\|x(t)-x_{1}(t)\right\|\|x(t)\| \\
& <B\left\|y(t)-y_{1}(t)\right\|\|y(t)\| \\
& <C\left\|z(t)-z_{1}(t)\right\|\|z(t)\|
\end{aligned}
$$

We conclude from the results of Equations (50) and (52) that the iterative method used is stable. Then, we obtain the same in [31]:

$$
\begin{gathered}
x(t)=\sum_{u=0}^{n-1} \delta_{1}^{u} \frac{t^{u}}{u !}+\frac{1}{\Gamma(\kappa)} \int_{0}^{t}(t-\psi)^{\kappa-1}[z(\psi)+x(\psi) y(\psi)-a x(\psi)] d u, \\
y(t)=\sum_{u=0}^{n-1} \delta_{2}^{u} \frac{t^{u}}{u !}+\frac{1}{\Gamma(\kappa)} \int_{0}^{t}(t-\psi)^{\kappa-1}[1-b y(\psi)+x(\psi) x(\psi)] d u, \\
z(t)=\sum_{u=0}^{n-1} \delta_{3}^{u} \frac{t^{u}}{u !}+\frac{1}{\Gamma(\kappa)} \int_{0}^{t}(t-\psi)^{\kappa-1}[d-x(\psi)-c z(\psi)] d u,
\end{gathered}
$$




\section{Atangana-Baleanu-Caputo Sense}

Considering the system with an $\mathrm{ABC}$ fractional order derivative according to the methodology mentioned in $[23,26]$ :

$$
\begin{aligned}
& { }_{0}^{A B C} D_{t}^{\kappa} x(t)=z+x y-a x \\
& { }_{0}^{A B C} D_{t}^{\kappa} y(t)=1-b y-x x \\
& { }_{0}^{A B C} D_{t}^{\kappa} z(t)=d-x-c z
\end{aligned}
$$

with initial conditions $x(0)=n_{1} \geq 0, y(0)=n_{2} \geq 0, z(0)=n_{3} \geq 0$

Solution: We apply the Laplace transformation (4) to Equation (56), we have

$$
\frac{R(\kappa)}{1-\kappa} \frac{s^{\kappa} \tilde{x}(s)-s^{\kappa-1} x(0)}{s^{\kappa}+\frac{\kappa}{1-\kappa}}=\mathcal{L}\{z(t)+x(t) y(t)-a x(t)\}
$$

Simplifying the above equation with taking initial conditions

$$
\tilde{x}(s)=\frac{x(0)}{s}+\frac{(1-\kappa) s^{\kappa}+\kappa}{R(\kappa) s^{\kappa}} \mathcal{L}\{z(t)+x(t) y(t)+a x(t)\},
$$

Then, we have

$$
x(t)=n_{1}+\mathcal{L}^{-1}\left\{\frac{(1-\kappa) s^{\kappa}+\kappa}{R(\kappa) s^{\kappa}} \mathcal{L}\{z(t)+x(t) y(t)+a x(t)\}\right\},
$$

Similarly to Equations (57) and (58), we have

$$
\begin{gathered}
y(t)=n_{2}+\frac{(1-\kappa)}{R(\kappa) s}+\frac{\kappa}{R(\kappa) s(\kappa)}-\mathcal{L}^{-1}\left\{\frac{(1-\kappa) s^{\kappa}+\kappa}{R(\kappa) s^{\kappa}} \mathcal{L}\{b y(t)+x(t) x(t)\}\right\}, \\
z(t)=n_{3}+\frac{(1-\kappa)}{R(\kappa) s}+\frac{\kappa}{R(\kappa) s(\kappa)}-\mathcal{L}^{-1}\left\{\frac{(1-\kappa) s^{\kappa}+\kappa}{R(\kappa) s^{\kappa}} \mathcal{L}\{x(t)+c z(t)\}\right\},
\end{gathered}
$$

Here, we select a operator that is of linear type as

$$
[\mathfrak{j}(\mathfrak{t} ; \mathfrak{p})]=\mathcal{L}\left[\phi_{j}(t ; p)\right], j=1,2,3 .
$$

Next, we describe the model below:

$$
\begin{gathered}
N\left[\phi_{1}(t ; p)\right]=\mathcal{L}\left[\phi_{1}(t ; p)\right]-n_{1}-\frac{(1-\kappa) s^{\kappa}+\kappa}{R(\kappa) s^{\kappa}} \mathcal{L}\left\{\phi_{3}-\phi_{1} \phi_{2}+a \phi_{1}\right\} \\
N\left[\phi_{2}(t ; p)\right]=\mathcal{L}\left[\phi_{2}(t ; p)\right]-n_{2}-\frac{(1-\kappa) s^{\kappa}+\kappa}{R(\kappa) s^{\kappa}} \mathcal{L}\left\{b \phi_{2}+\phi_{1}^{2}\right\} \\
N\left[\phi_{3}(t ; p)\right]=\mathcal{L}\left[\phi_{3}(t ; p)\right]-n_{3}-\frac{(1-\kappa) s^{\kappa}+\kappa}{R(\kappa) s^{\kappa}} \frac{1}{s^{\kappa}} \mathcal{L}\left\{\phi_{1}+c \phi_{3}\right\}
\end{gathered}
$$

This is the so-called zeroth-order deformation is presented by:

$$
(1-p)\left[\mathfrak{j}(\mathfrak{t} ; \mathfrak{p})-\mathfrak{u}_{\mathcal{o}}(\mathfrak{t})\right]=p \hbar N\left[\phi_{j}(t ; p)\right], j=1,2,3
$$




$$
\phi_{j}(t ; 0)=u_{0}(t), \phi_{j}(t ; 1)=u(t), j=1,2,3
$$

The equations of the mth-order deformation are presented by

$$
\begin{aligned}
& \mathcal{L}\left\{x_{m}(t)-P_{m} x_{m-1}(t)\right\}=\hbar S_{m}\left(x_{m-1}, t\right) \\
& \mathcal{L}\left\{y_{m}(t)-P_{m} y_{m-1}(t)\right\}=\hbar S_{m}\left(y_{m-1}, t\right) \\
& \mathcal{L}\left\{z_{m}(t)-P_{m} z_{m-1}(t)\right\}=\hbar S_{m}\left(z_{m-1}, t\right)
\end{aligned}
$$

Use the inverse Laplace to transform the Equations (69)-(71), and we obtain

$$
\begin{aligned}
& x_{m}(t)=P_{m} x_{m-1}(t)+\hbar S_{m}\left(x_{m-1}, t\right) \\
& y_{m}(t)=P_{m} y_{m-1}(t)+\hbar S_{m}\left(y_{m-1}, t\right) \\
& z_{m}(t)=P_{m} z_{m-1}(t)+\hbar S_{m}\left(z_{m-1}, t\right)
\end{aligned}
$$

where

$$
\begin{gathered}
S_{m}\left(x_{m-1}, t\right)=\mathcal{L}\left[x_{m-1}(t)\right]-\left(1-P_{m}\right) n_{1}+\frac{(1-\kappa) s^{\kappa}+\kappa}{R(\kappa) s^{\kappa}} \mathcal{L}\left\{z_{m-1}+H_{m-1}-a x_{m-1}\right\} \\
S_{m}\left(y_{m-1}, t\right)=\mathcal{L}\left[y_{m-1}(t)\right]-\left(1-P_{m}\right)\left(n_{2}+\frac{(1-\kappa)}{R(\kappa)}+\frac{\kappa \epsilon^{\kappa}}{\Gamma(\kappa+1)}\right)-\frac{(1-\kappa) s^{\kappa}+\kappa}{R(\kappa) s^{\kappa}} \mathcal{L}\left\{b y_{m-1}+K_{m-1}\right\} \\
S_{m}\left(z_{m-1}, t\right)=\mathcal{L}\left[z_{m-1}(t)\right]-\left(1-P_{m}\right)\left(n_{3}+\frac{d(1-\kappa)}{R(\kappa)}+\frac{d \kappa t^{\kappa}}{\Gamma(\kappa+1)}\right)-\frac{(1-\kappa) s^{\kappa}+\kappa}{R(\kappa) s^{\kappa}} \mathcal{L}\left\{x_{m-1}+c z_{m-1}\right\}
\end{gathered}
$$

The mth-order deformation of the system is specified as

$$
\begin{gathered}
x_{m}(t)=\left(P_{m}+\hbar\right) x_{m-1}-\hbar\left(1-P_{m}\right) n_{1}+\hbar \mathcal{L}^{-1}\left\{\frac{(1-\kappa) s^{\kappa}+\kappa}{R(\kappa) s^{\kappa}} \mathcal{L}\left[z_{m-1}+H_{m}-a x_{m-1}\right]\right\} \\
\begin{array}{r}
y_{m}(t)=\left(P_{m}+\hbar\right) y_{m-1}-\hbar\left(1-P_{m}\right)\left(n_{2}+\frac{(1-\kappa)}{R(\kappa)}+\frac{t^{\kappa}}{R(\kappa) \Gamma \kappa+1}\right)- \\
\hbar \mathcal{L}^{-1}\left\{\frac{(1-\kappa) s^{\kappa}+\kappa}{R(\kappa) s^{\kappa}} \mathcal{L}\left[b y_{m-1}+K_{m}\right]\right\} \\
z_{m}(t)=\left(P_{m}+\hbar\right) z_{m-1}-\hbar\left(1-P_{m}\right)\left(n_{3}+\frac{d(1-\kappa)}{R(\kappa)}+\frac{d \kappa t^{\kappa}}{\Gamma \kappa+1}\right) \\
-\hbar \mathcal{L}^{-1}\left\{\frac{(1-\kappa) s^{\kappa}+\kappa}{R(\kappa) s^{\kappa}} \mathcal{L}\left[x_{m-1}+c z_{m-1}\right]\right\}
\end{array}
\end{gathered}
$$

where

$$
H_{m}=\frac{1}{\Gamma(m+1)}\left[\frac{d^{m}}{d p^{m}} N\left[\left(p \phi_{1}(t ; p)\right)\left(p \phi_{2}(t ; p)\right)\right]\right]_{p=0^{\prime}}
$$




$$
K_{m}=\frac{1}{\Gamma(m+1)}\left[\frac{d^{m}}{d p^{m}} N\left[\left(p \phi_{1}(t ; p)\right)\left(p \phi_{1}(t ; p)\right)\right]\right]_{p=0^{\prime}}
$$

Finally, the solutions of Equations (56)-(58) are given as

$$
x(t)=\sum_{m=0}^{\infty} x_{m}(t), y(t)=\sum_{m=0}^{\infty} y_{m}(t), z(t)=\sum_{m=0}^{\infty} z_{m}(t)
$$

Models (56)-(58) solution can be obtained with Equation (4). Systems (56)-(58) are similar to the Volterra form in the Atangana-Baleanu sense. With the iterative scheme, we get

$$
\begin{gathered}
x_{n+1}(t)=\frac{1-\kappa}{R(\kappa)}\left\{z_{n}(t)+x_{n}(t) y_{n}(t)-a x_{n}(t)\right\}+ \\
\frac{\kappa}{R(\kappa) \Gamma(\kappa)} \int_{0}^{t}(t-\psi)^{\kappa-1}\left\{z_{n}(\psi)+x_{n}(\psi) y_{n}(\psi)-a x_{n}(\psi)\right\} d \psi, \\
y_{n+1}(t)=\frac{1-\kappa}{R(\kappa)}\left\{1-b y_{n}(t)-x_{n}(t) x_{n}(t)\right\}+ \\
\frac{\kappa}{R(\kappa) \Gamma(\kappa)} \int_{0}^{t}(t-\psi)^{\kappa-1}\left\{1-b y_{n}(\psi)-x_{n}(\psi) x_{n}(\psi)\right\} d \psi, \\
z_{n+1}(t)=\frac{1-\kappa}{R(\kappa)}\left\{d-x_{n}(t)-c z_{n}(t)\right\}+ \\
\frac{\kappa}{R(\kappa) \Gamma(\kappa)} \int_{0}^{t}(t-\psi)^{\kappa-1}\left\{d-x_{n}(\psi)-c z_{n}(\psi)\right\} d \psi
\end{gathered}
$$

Theorem 2. We prove the existence and uniqueness of the solution using a Picard-Lindelof approach.

Proof. The following operator is considered:

$$
\begin{array}{r}
\Xi_{1}(t, \varsigma)=z(t)+x(t) y(t)-a x(t) \\
\Xi_{2}(t, \varsigma)=1-b y(t)-x(t) x(t) \\
\Xi_{3}(t, \varsigma)=d-x(t)-c z(t)
\end{array}
$$

Let

$$
\begin{aligned}
& \Omega_{1}=\sup \left\|\gamma_{\epsilon, k_{1}} \Xi_{1}(t, \varsigma)\right\| ; \\
& \Omega_{2}=\sup \left\|\gamma_{\epsilon, k_{2}} \Xi_{2}(t, \varsigma)\right\| ; \\
& \Omega_{3}=\sup \left\|\gamma_{\epsilon, k_{3}} \Xi_{3}(t, \varsigma)\right\| ;
\end{aligned}
$$

where

$$
\begin{aligned}
& \gamma_{\epsilon, k_{1}}=|t-a, t+a| \times\left[\vartheta-k_{1}, \vartheta+k_{1}\right]=\epsilon_{1} \times k_{1} \\
& \gamma_{\epsilon, k_{2}}=|t-a, t+a| \times\left[\vartheta-k_{2}, \vartheta+k_{2}\right]=\epsilon_{1} \times k_{2} \\
& \gamma_{\epsilon, k_{3}}=|t-a, t+a| \times\left[\vartheta-k_{3}, \vartheta+k_{3}\right]=\epsilon_{1} \times k_{3}
\end{aligned}
$$


Considering the Picards operator, we have

$$
\vartheta: \gamma\left(\epsilon_{1}, k_{1}, k_{2}, k_{3}\right) \rightarrow \gamma\left(\epsilon_{1}, k_{1}, k_{2}, k_{3}\right)
$$

defined as follows:

$$
\vartheta \Omega(t)=\Omega_{0}(t)_{\Delta}(t, \Omega(t)) \frac{1-\kappa}{R(\kappa)}+\frac{\kappa}{R(\kappa) \Gamma(\kappa)} \int_{0}^{t}(t-\psi)^{\kappa-1} \Delta(\psi, \Omega(\psi)) d \psi,
$$

where

$$
\Omega(t)=\{G(t), X(t), I(t)\}=\left\{g_{1}, g_{2}, g_{3}\right\}, \text { and } \Delta(t, \Omega(t))=\left\{\Xi_{1}(t, \vartheta(t)), \Xi_{2}(t, \vartheta(t)), \Xi_{1}(t, \vartheta(t))\right\} \text {. }
$$

Now, we presume that all solutions are bound in a certain amount of time

$$
\begin{array}{r}
\|\Omega(t)\|_{\infty} \leq \max \left\{k_{1}, k_{2}, k_{3}\right\}, \\
\left\|\Omega(t)-\Omega_{0}(t)\right\|=\| \Delta(t, \Omega(t)) \frac{1-\kappa}{R(\kappa)}+\frac{\kappa}{R(\kappa) \Gamma(\kappa)} \int_{0}^{t}(t-\psi)^{\kappa-1} \Delta(\psi, \Omega(\psi) d \psi \| \\
\leq \frac{1-\kappa}{R(\kappa)}\|\Delta(t, \Omega(t))\|+\frac{\kappa}{R(\kappa) \Gamma(\kappa)} \int_{0}^{t}(t-\psi)^{\kappa-1} \| \Delta(\psi, \Omega(\psi) \| d \psi \\
\leq \frac{1-\kappa}{R(\kappa)} X=\max \left\{k_{1}, k_{2}, k_{3}\right\}+\frac{\kappa}{R(\kappa)} \xi \vartheta^{\kappa} \leq \vartheta \xi \leq k=\max \left\{k_{1}, k_{2}, k_{3}\right\}
\end{array}
$$

Here, we request that $\vartheta<\frac{k}{\widetilde{\zeta}}$ Then, we obtain

$$
\begin{gathered}
\left\|\vartheta \Omega_{1}-\vartheta \Omega_{2}\right\|_{\infty}=\sup \|_{t \in \varepsilon}\left|\Omega_{1}-\Omega_{2}\right|, \\
\left\|\vartheta \Omega_{1}-\vartheta \Omega_{2}\right\|=\|\left\{\left(t, \Omega_{1}(t)\right)-\Delta\left(t, \Omega_{2}(t)\right)\right\} \frac{1-\kappa}{R(\kappa)} \\
+\frac{\kappa}{R(\kappa) \Gamma(\kappa)} \int_{0}^{t}(t-\psi)^{\kappa-1}\left\{\Delta\left(\psi, \Omega_{1}(t)\right)-\Delta\left(\psi, \Omega_{2}(t)\right)\right\} d \psi \|, \\
\leq \frac{1-\kappa}{R(\kappa)}\left\|\Delta\left(\psi, \Omega_{1}(t)\right)-\Delta\left(\psi, \Omega_{2}(t)\right)\right\|+\frac{\kappa}{R(\kappa) \Gamma(\kappa)} \int_{0}^{t}(t-\psi)^{\kappa-1}\left\{\Delta\left(\psi, \Omega_{1}(t)\right)-\| \Delta\left(\psi, \Omega_{2}(t)\right)\right\} \| d \psi, \\
\left.\left.\leq \frac{1-\kappa}{R(\kappa)} \omega\left\|\Omega_{1}(t)-\Omega_{2}(t)\right\|+\frac{\kappa \omega}{R(\kappa) \Gamma(\kappa)} \int_{0}^{t}(t-\psi)^{\kappa-1} \| \Omega_{1}(t)-\Omega_{2}(t)\right)\right) \| d \psi, \\
\leq\left\{\frac{1-\kappa}{R(\kappa)} \omega+\frac{\kappa \omega \vartheta \kappa}{R(\kappa) \Gamma(\kappa)}\right\}\left\|\Omega_{1}(t)-\Omega_{2}(t)\right\| d \psi, \\
\leq \vartheta \omega\left\|\Omega_{1}(t)-\Omega_{2}(t)\right\|,
\end{gathered}
$$

with $\omega$ less than 1 . Since $\Omega$ is a contraction, we obtain $\theta \omega<1$, so the specified $\vartheta$ operator is also a contraction. The Atangana-Baleanu fractional integral numerical approximation [27] using the Adams-Moulton rule is given by

$$
\psi_{t}^{\kappa}\left[g\left(t_{n+1}\right)\right]=\frac{1-\kappa}{R(\kappa)} \frac{g\left(t_{n+1}-g\left(t_{n}\right)\right)}{2}+\frac{\kappa}{\Gamma(\kappa)} \sum_{k=0}^{\infty}\left[\frac{g\left(t_{k+1}-g\left(t_{k}\right)\right)}{2}\right] b_{k}^{\kappa}
$$

where $b_{k}^{\kappa}=(k+1)^{1-\kappa}-(k)^{1-\kappa}$. Hence, it shows that existence and uniqueness of the solution for the dynamical finance system. We have the following generalized solution with the iterative method: 


$$
\begin{gathered}
x_{(n+1)}(t)-x_{(n)}(t)=x_{0}^{n}(t)+\left\{\frac { 1 - \kappa } { R ( \kappa ) } \left[\left(\frac{z_{(n+1)}(t)-z_{(n)}(t)}{2}\right)+\left(\frac{x_{(n+1)}(t)-x_{(n)}(t)}{2}\right)\right.\right. \\
\left.\left.\left(\frac{y_{(n+1)}(t)-y_{(n)}(t)}{2}\right)-a\left(\frac{x_{(n+1)}(t)-x_{(n)}(t)}{2}\right)\right]\right\}+\frac{\kappa}{R(\kappa)} \sum_{k=0}^{\infty}(k+1)^{d} 1-\kappa\left[\left(\frac{z_{(k+1)}(t)-z_{(k)}(t)}{2}\right)\right. \\
\left.+\left(\frac{x_{(k+1)}(t)-x_{(k)}(t)}{2}\right)\left(\frac{y_{(k+1)}(t)-y_{(k)}(t)}{2}\right)-a\left(\frac{x_{(k+1)}(t)-x_{(k)}(t)}{2}\right)\right] \\
y_{(n+1)}(t)-y_{(n)}(t)=y_{0}^{n}(t)+\left\{\frac { 1 - \kappa } { R ( \kappa ) } \left[1-b\left(\frac{y_{(n+1)}(t)-y_{(n)}(t)}{2}\right)-\left(\frac{x_{(n+1)}(t)-x_{(n)}(t)}{2}\right)\right.\right. \\
\left.\left.\left(\frac{x_{(n+1)}(t)-x_{(n)}(t)}{2}\right)\right]\right\}+\frac{\kappa}{R(\kappa)} \sum_{k=0}^{\infty}(k+1)^{1-\kappa}\left[1-b\left(\frac{y_{(k+1)}(t)-y_{(k)}(t)}{2}\right)-\left(\frac{x_{(k+1)}(t)-x_{(k)}(t)}{2}\right)\right. \\
\left.\left(\frac{x_{(k+1)}(t)-x_{(k)}(t)}{2}\right)\right] \\
z_{(n+1)}(t)-z_{(n)}(t)=z_{0}^{n}(t)+\left\{\frac{1-\kappa}{R(\kappa)}\left[d-\left(\frac{x_{(n+1)}(t)-x_{(n)}(t)}{2}\right)-c\left(\frac{z_{(n+1)}(t)-z_{(n)}(t)}{2}\right)\right]\right\}+ \\
\kappa \\
R(\kappa) \\
\sum_{k=0}^{\infty}(k+1)^{1-\kappa}\left[d-\left(\frac{x_{(k+1)}(t)-x_{(k)}(t)}{2}\right)-c\left(\frac{z_{(k+1)}(t)-z_{(k)}(t)}{2}\right)\right]
\end{gathered}
$$

\section{Numerical Results and Discussion}

The $\mathrm{ABC}$ derivative has been used to present the theoretical solution of the fractional-order model consisting of a nonlinear system of the fractional differential equation. In this model, we represent $x(t), y(t)$ and $z(t)$ are interest rate, investment demand, and price exponent with initial conditions $x(0)=0.1, y(0)=4$ and $z(0)=0.5$, while the parameter $a$ is for savings, $\mathrm{b}$ is to cost per investment, and $c$ is the commercial markets demand elasticity with $a=3, b=0.1$, and $c=1$ are given in [30,32]. By utilizing Caputo and $\mathrm{ABC}$ fractional derivative, the numerical results of interest rate, investment demand, and price exponent for various fractional estimations of $\eta$ are acquired. Figures $1-3$ refer to the graphical solution of the finance system with the Caputo derivative of the finance system. Within this figure, we noticed that interest rate, investment demand, and the price exponent have more degree of freedom as contrasted with ordinary derivatives. From Figures 4-6, we use ABC fractional-order derivative of the financial system, we effectively have seen that interest rate, investment demand, and price exponent rates are the better estimations compared with ordinary derivatives. Figures 7-10 present the comparison of Caputo derivative and $A B C$ derivative for the finance system. It should be observed that the behavior of the finance system is almost the same but $A B C$ derivative presents more convenient and comfortable behavior in a system for closed-loop design. Caputo and $A B C$ fractional derivatives are increasing or decreasing in the relationship between these variables. From Figures 1-10, remarkable responses are obtained from the developed model for compartments by taking non-integer fractional parameter values. Numerical results show that the system keeps the $\eta$ chaotic motion. The interest rate begins to rise according to initial conditions as investment demand and price exponent begin to fall, which shows the financial system's actual macroeconomic behavior. It is observed here that a complex chaotic fractional system provides more appropriate and reliable results compared to time integer parameters for non-integer time-fractional parameters. In this system, we add parameter $d$ to develop the new financial stable model which is the critical minimum interest rate. In order to observe the impact of factors on the mechanics of the fractional-order model, different numerical ways can be observed in Figures 11-13. These simulations reveal a change in the value of a critical minimum interest rate of the model. We see that decreasing the critical minimum interest rate decreases the price of the exponent and the investment demand becomes high. Due to increasing the investment demand, our economy will become stronger. 


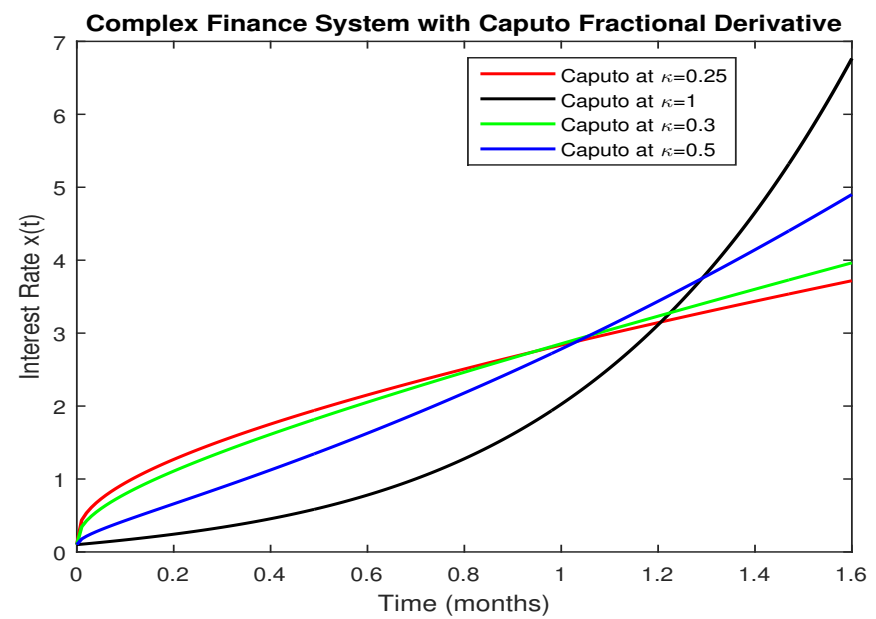

Figure 1. $x(t)$ interest rate with Caputo fractional derivative.

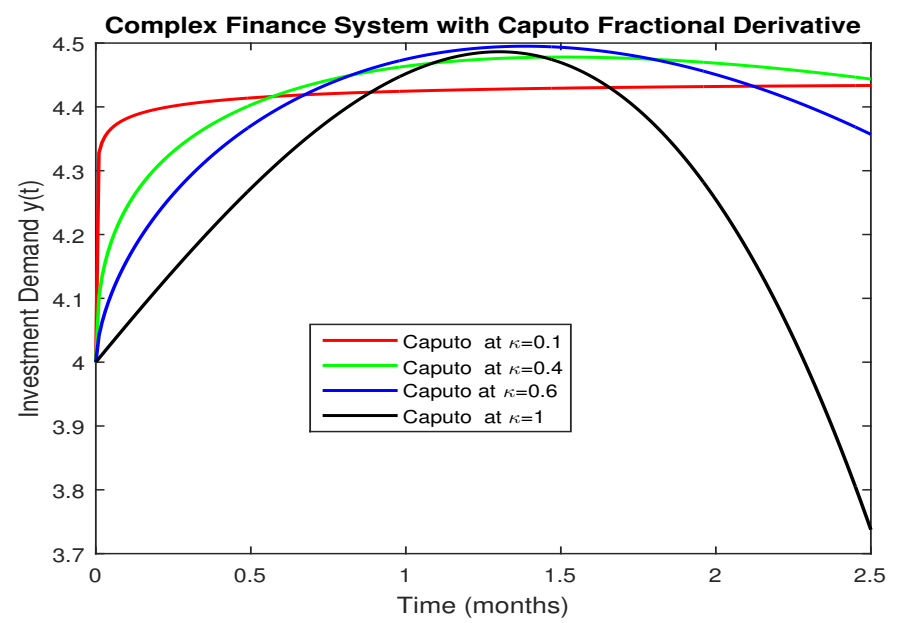

Figure 2. $y(t)$ investment demand with Caputo fractional derivative.

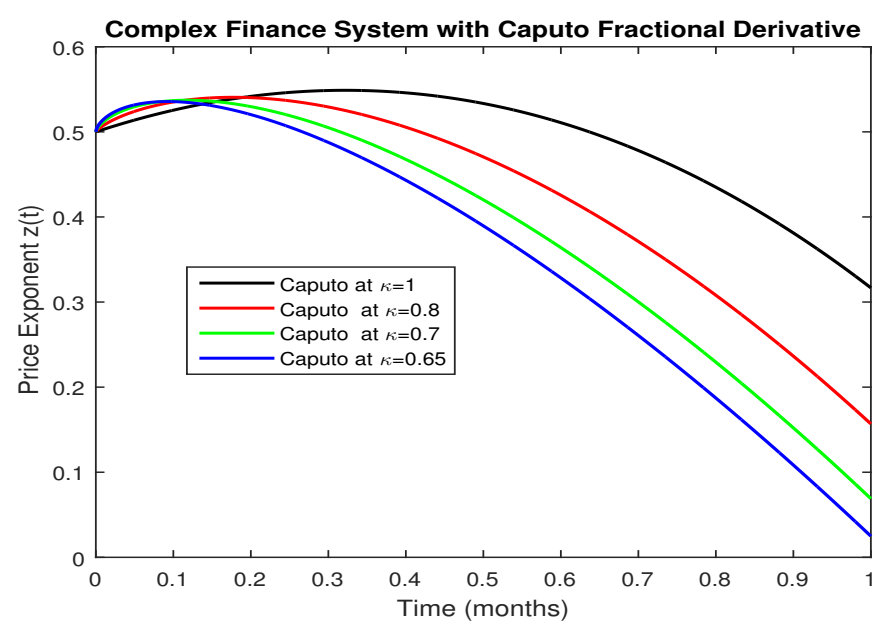

Figure 3. $z(t)$ price exponent with Caputo fractional derivative. 


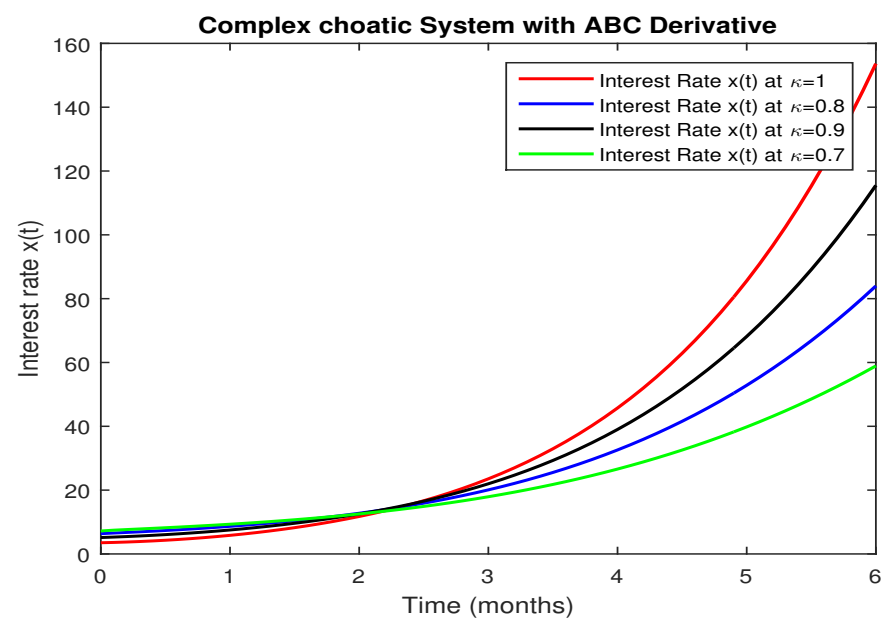

Figure 4. $x(t)$ interest rate with $\mathrm{ABC}$ derivative.

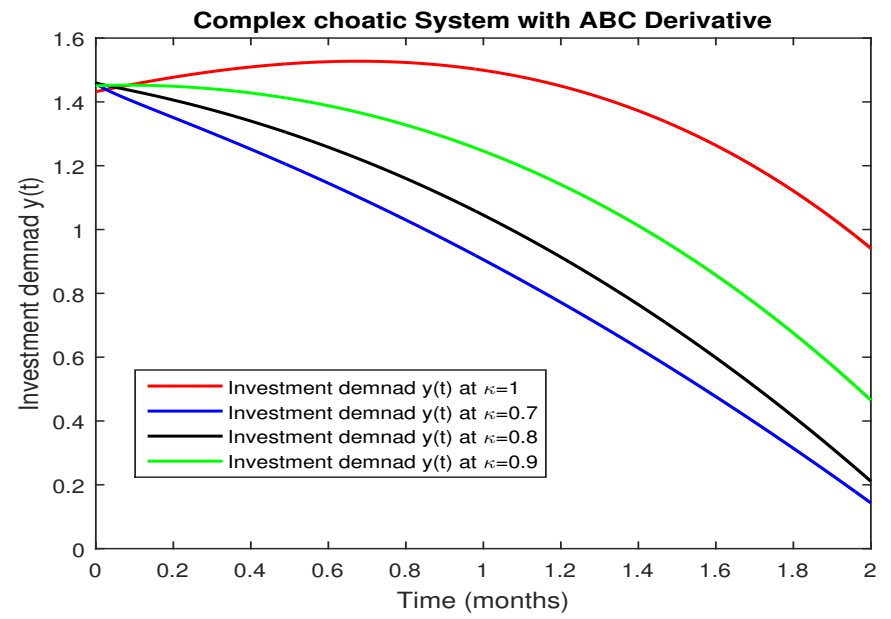

Figure 5. $y(t)$ investment demand with $\mathrm{ABC}$ derivative.

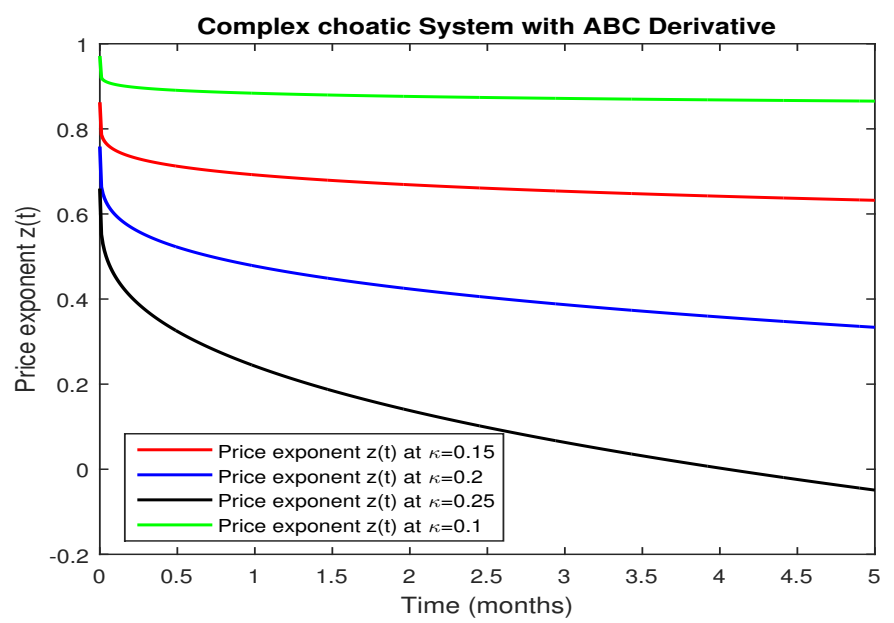

Figure 6. $z(t)$ price exponent with $\mathrm{ABC}$ derivative. 


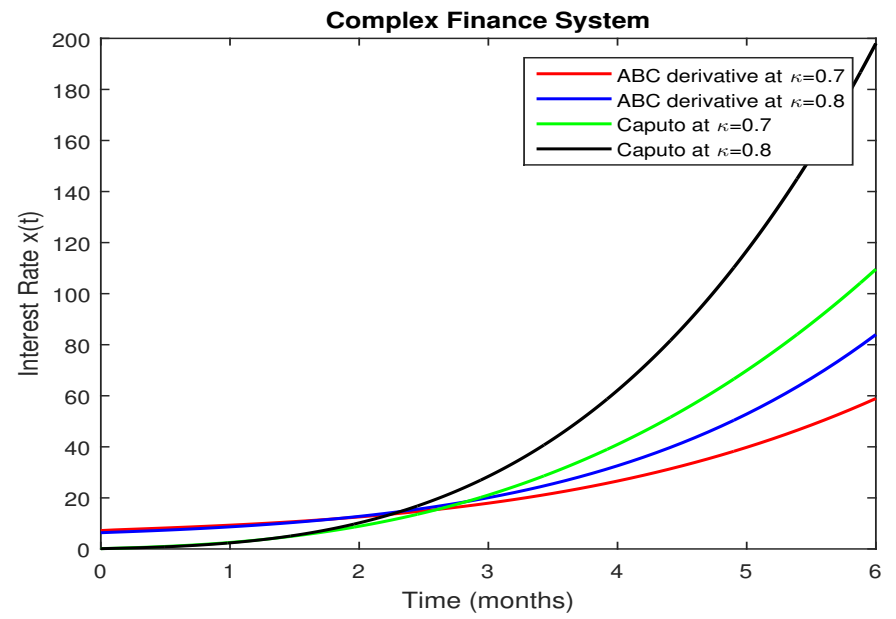

Figure 7. $x(t)$ interest rate with Caputo and $\mathrm{ABC}$ derivative.

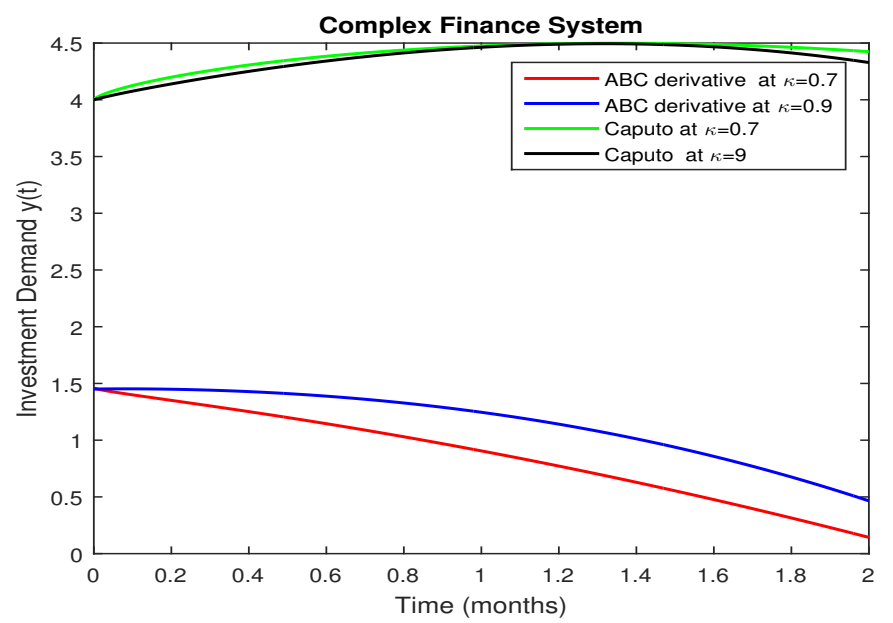

Figure 8. $y(t)$ investment demand with Caputo and $\mathrm{ABC}$ derivative.

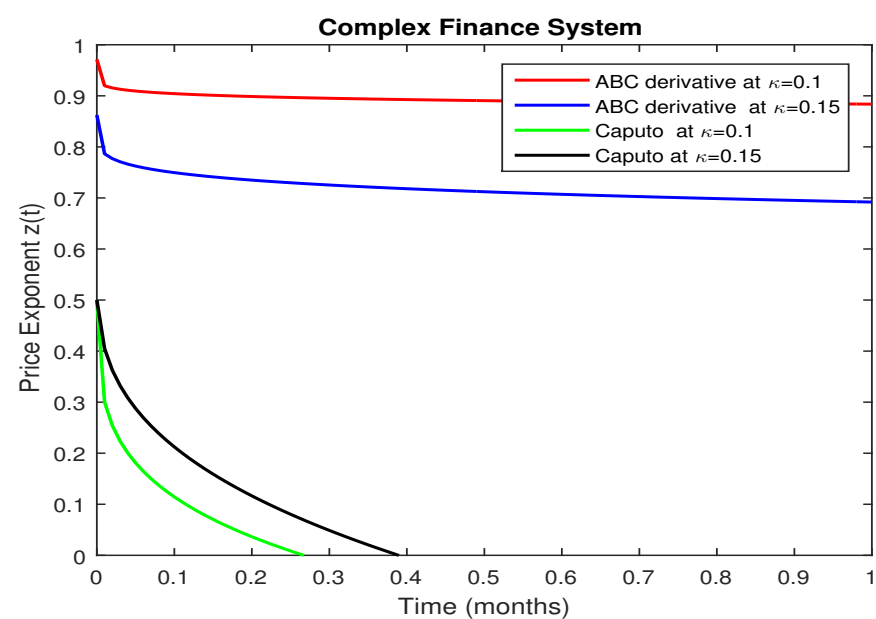

Figure 9. $z(t)$ price exponent with Caputo and $\mathrm{ABC}$ derivative. 


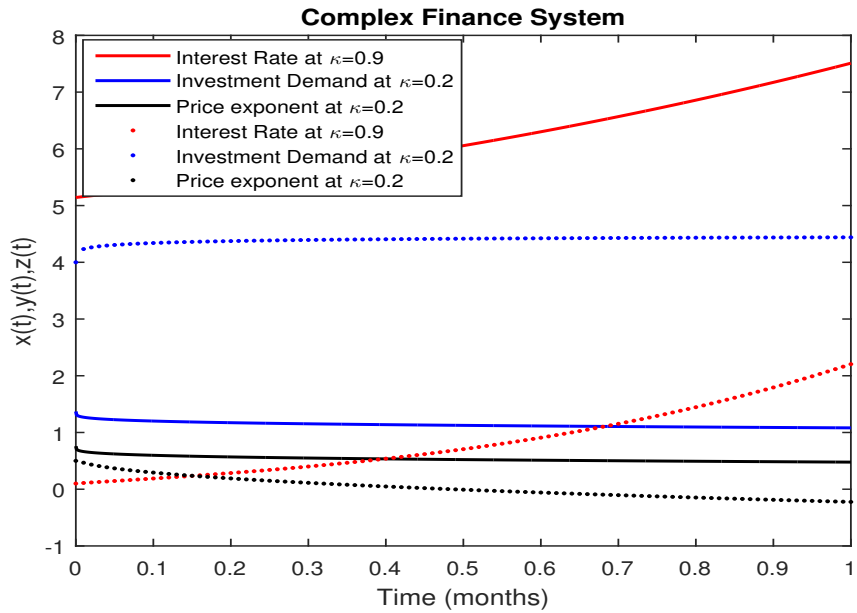

Figure 10. $z(t)$ price exponent with Caputo and $\mathrm{ABC}$ derivative.

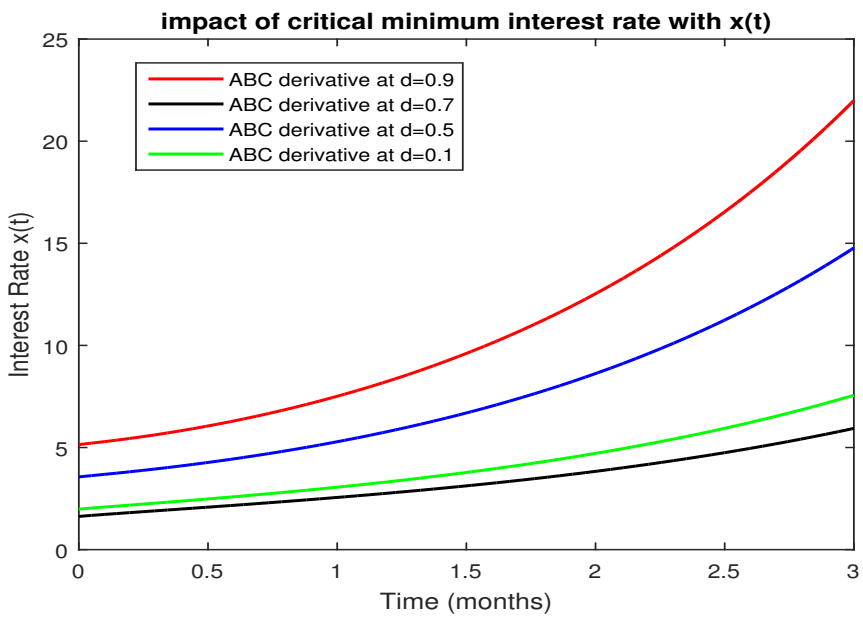

Figure 11. Impact of critical minimum interest rate with $x(t)$.

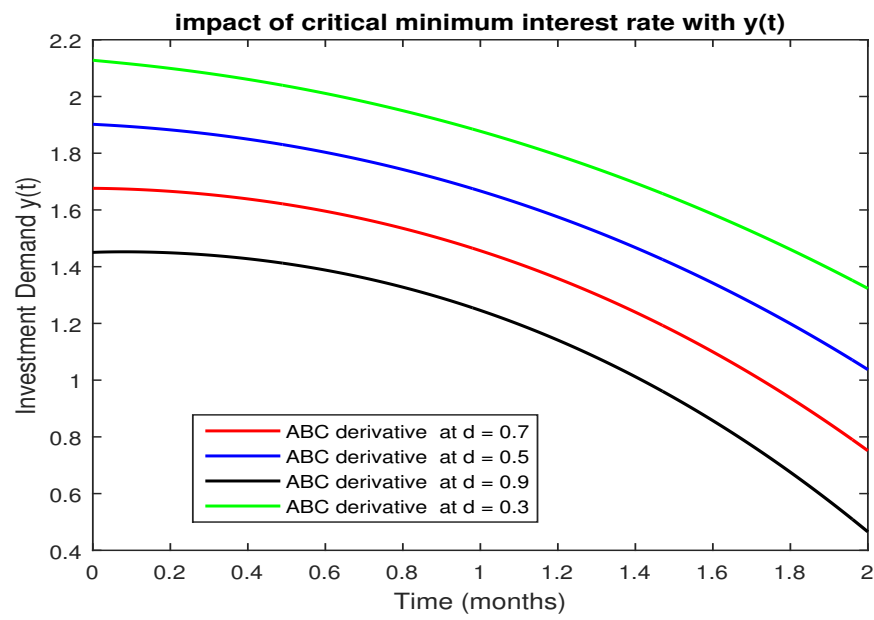

Figure 12. Impact of critical minimum interest rate with $y(t)$. 


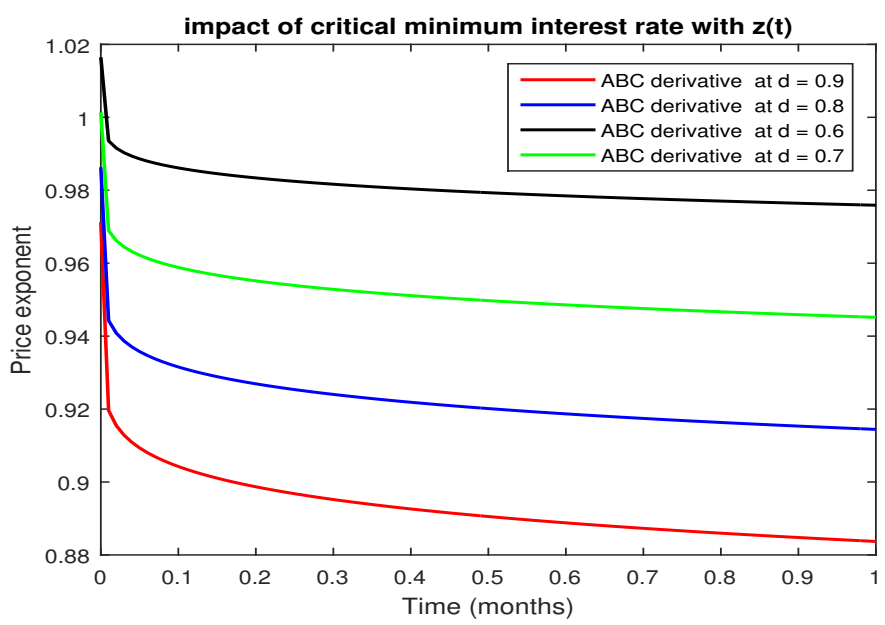

Figure 13. Impact of critical minimum interest rate with $z(t)$.

\section{Conclusions}

This paper uses a dynamic chaotic fractional order model with an $\mathrm{ABC}$ derivative to conduct the economic system. The basis of this fractional model consists of exponentially decreasing non-singular kernels that appear in the derivation of the ABC. The financial model is presented with theoretical and numerical investigation. This demonstrates the regulation of the economic system's critical minimum interest rate. In order to control the economic system, we are discussing a fractional order financing model. The modified model with $A B C$ derivative shows a good financial system control agreement. The model offers the effect of evaluating numerical results on a critical minimum interest rate. Graphical representation shows the impact on the amount of critical minimum interest rate for variables with time. We can observe $\kappa=1$ revealing more absorbing characteristics by numerical simulation using $\mathrm{ABC}$ non-integer order derivative. For interest rate, investment demand and price exponent, the concept of this research provides important results. Therefore, we conclude that the $\mathrm{ABC}$ derivative is useful to control and maintain the finance system to overcome the risk factors. The interest rate begins to rise according to initial conditions as investment demand and price exponent begin to fall, which shows the actual macroeconomic behavior of the financial system. It is observed here that the complex chaotic fractional system provides more appropriate and reliable results as compared to time integer parameters for non-integer time-fractional parameters.

Author Contributions: Conceptualization, M.F. and A.A. (Ali Akgül); Methodology, A.A. (Ali Akgül); Software, S.I.; Validation, A.H., A.A. (Aqeel Ahmad) and D.B.; Formal Analysis, D.B.; Investigation, A.A. (Aqeel Ahmad); Resources, A.A. (Aqeel Ahmad); Data Curation, M.F.; Writing-Original Draft Preparation, M.F.; Writing-Review \& Editing, M.F.; All authors have read and agreed to the published version of the manuscript.

Funding: This research received no external funding .

Conflicts of Interest: The authors declare no conflict of interest.

\section{References}

1. Neumaier, A. Mathematical Modelling. Available online: http://www.mat.univie.ac.at/ neum/ms/model. pdf (accessed on 18 January 2011).

2. Merton, R.C.; Simons, R.V.; Wilkie, A.D. Influence of mathematical models in finance on practice: Past, present and future. Philos. Trans. Phys. Sci. Eng. 1994, 347, 451-463.

3. Karatzas, I. Lectures on the Mathematics of Finance 8; American Mathematical Society: Providence, RI, USA, 1997.

4. Hilfer, R. Applications of Fractional Calculus in Physics; World Scientific: Singapore, 2000; pp. 87-130.

5. Kumar, D.; Singh, J.; Baleanu, D. Modified Kawahara equation within a fractional derivative with non-singular kernel. Therm. Sci. 2017, 22, 789-796. 
6. Kumar, D.; Singh, J.; Baleanu, D.; Sushila. Analysis of regularized long-wave equation associated with a new fractional operator with Mittag-Leffler type kernel. Physica A 2018, 492, 155-167. [CrossRef]

7. Baleanu, D.; Jajarmi, A.; Hajipour, M.; A new formulation of the fractional optimal control problems involving Mittag-Leffler nonsingular kernel. J. Optim. Theory Appl. 2017, 175, 718-737. [CrossRef]

8. Baleanu, D.; Jajarmi, A.; Asad, J.H.; Blaszczyk, T. The motion of a bead sliding on a wire in fractional sense. Acta Phys. Pol. A 2017, 131, 1561-1564. [CrossRef]

9. Jajarmi, A.; Hajipour, M.; Baleanu, D. New aspects of the adaptive synchronization and hyperchaos suppression of a financial model. Chaos Solitons Fractals 2017, 99, 285-296. [CrossRef]

10. Kilbas, A.A.; Srivastava, H.M.; Trujillo, J.J. Theory and Applications of Fractional Differential Equations; Elsevier: Amsterdam, The Netherlands, 2006. [CrossRef]

11. Baleanu, D.; Guvenc, Z.B.; Machado, J.A.T. New Trends in Nanotechnology and Fractional Calculus Applications; Springer: Dordrecht, The Netherlands, 2000. [CrossRef]

12. Yang, X.J.; Srivastava, H.M.; Machado, J.A.T. A new fractional derivative without singular kernel: Application to the modelling of the steady heat flow. Therm. Sci. 2016, 20, 753-756. [CrossRef]

13. Caputo, M.; Fabrizio, M. A new definition of fractional derivative without singular kernel. Prog. Fract. Differ. Appl. 2015, 1, 73-85.

14. Atangana, A. On the new fractional derivative and application to nonlinear Fisher's reaction diffusion equation. Appl. Math. Comput. 2016, 273, 948-956.

15. Panda, S.K.; Karapınar, E.; Atangana A. A numerical schemes and comparisons for fixed point results with applications to the solutions of Volterra integral equations in dislocated extended b a metric space. Alex. Eng. J. 2020, 59, 815-827. [CrossRef]

16. Yavuz, M.; Özdemir, N. European vanilla option pricing model of fractional order without singular kernel. Fractal Fract. 2018, 2, 3. [CrossRef]

17. Keten, A.; Yavuz, M.; Baleanu, D. Nonlocal Cauchy problem via a fractional operator involving power kernel in Banach Spaces. Fractal Fract. 2019, 3, 27. [CrossRef]

18. Yavuz, M.; Abdeljawad, T. Nonlinear regularized long-wave models with a new integral transformation applied to the fractional derivative with power and Mittag-Leffler kernel. Adv. Differ. Equ. 2020, 2020, 367. [CrossRef]

19. Yavuz, M.; Ozdemir, N.; Baskonus, H.M. Solutions of partial differential equations using the fractional operator involving Mittag-Leffler kernel. Eur. Phys. J. Plus 2018, 133, 215. [CrossRef]

20. Gao, W.; Baskonus, H.M.; Shi, L. New investigation of Bats-Hosts Reservoir-People coronavirus model and apply to 2019-nCoV system. Adv. Differ. Equ. 2020, 2020, 1-11.

21. Gao, W.; Veeresha, P.; Prakasha, D.G.; Baskonus, H.M. Novel dynamical structures of 2019-nCoV with nonlocal operator via powerful computational technique. Biology 2020, 9, 107. [CrossRef]

22. Gao, W.; Veeresha, P.; Baskonus, H.M.; Prakasha, D.G.; Kumar, P. A new study of unreported cases of 2019-nCOV epidemic outbreaks. Chaos Solitons Fractals 2020, 138, 109929. [CrossRef]

23. MoralesDelgadoa, V.F.; Aguilarb, J.F.; Taneco, M.A.; Escobar, R.F. Mathematical modeling of the smoking dynamics using fractional differential equations with local and nonlocal kernel. J. Nonlinear Sci. Appl. 2018, 11, 994-1014. [CrossRef]

24. Atangana A.; Baleanu D. New fractional derivatives with nonlocal and non Singular Kernel: Theory and Application to Heat Transfer Model. Therm. Sci. 2016, 20, 763-769. [CrossRef]

25. Kumar, S.; Kumar, A.; Argyros, I. K. A new analysis for the Keller-Segel model of fractional order. Numer. Algorithms 2017, 75, 213-228. [CrossRef]

26. Kumar, S.; Rashidi, M.M. New analytical method for gas dynamic equation arising in shock fronts. Comput. Phys. Commun. 2014, 185, 1947-1954. [CrossRef]

27. Odibat, Z.; Bataineh, A.S. An adaptation of homotopy analysis method for reliable treatment of strongly nonlinear problems: Construction of homotopy polynomials. Math. Methods Appl. Sci. 2015, 38, 991-1000.

28. Ma, J.H.; Chen, Y.S. Study for the bifurcation topological structure and the global complicated character of a kind of nonlinear finance system, I. Appl. Math. Mech. 2011, 22, 1240-1251. [CrossRef]

29. Singh, J.; Kumar, D.; Al Qurashi, M.; Baleanu, D. A new fractional model for giving up smoking dynamics. Adv. Differ. Equ. 2017, 2017, 88. [CrossRef]

30. Li, C.P.; Tao, C.X. On the fractional Adams method. Comput. Math. Appl. 2009, 58, 1573-1588. [CrossRef] 
31. Alkahtani, B.S.T. Chua's circuit model with Atangana Baleanu derivative with fractional order. Chaos Solitons Fractals 2016, 89, 547-551. [CrossRef]

32. Zhen, W.; Huang, X.; Shi, G. Analysis of nonlinear dynamics and chaos in a fractional-order financial system with time delay. Comput. Math. Appl. 2011, 62, 1531-1539. [CrossRef]

(C) 2020 by the authors. Licensee MDPI, Basel, Switzerland. This article is an open access article distributed under the terms and conditions of the Creative Commons Attribution (CC BY) license (http:/ / creativecommons.org/licenses/by/4.0/). 